\title{
Risk weighted alpha index - Analysis of the ASX50 index
}

\author{
Nipun Agarwala* \\ a RMIT University, Melbourne VIC 3083, Australia. \\ * Corresponding author's e-mail: nipun1@msn.com
}

H I G H L I G H T S:

1. A new alternate equity index method that extends on the previous alternate index models - merging alpha generation and risk reduction.

2. Daily data over the duration January 2002 to December 2012 was used from the Thomson Reuters Tick History database for this research.

3. Risk Weighted Alpha method attempts to pick stocks with lower stock price volatility, increasing alpha, basis the persistence of alpha on competitive advantage of the company in its industry, passive index that looks to outperform the market index by holding positive alpha stocks and with more active management a long/short portfolio can also be viably held.

4. Risk Weighted Alpha method is expected to outperform the underlying market capitalization ASX 50 index.

5. Additionally, two performance based methods (Treynor's Square and Appraisal Ratio) were also used to develop index portfolios to provide an alternative to the Risk Weighted Alpha method. However, the Risk Weighted Alpha method seems to perform better.

\section{Article History}

Received: $12-10-2013$

Accepted: 24-12-2013

Available online: 30-12-2013

Keywords:

Equity index models; Risk weighted alpha; Investment management; Portfolio construction.

JEL Classification:

G02; G11; G14; G15.

(C) 2013 The Authors. This is an open access article under the terms of the Creative Commons Attribution License 4.0, which allows use, distribution and reproduction in any medium, provided the original work is properly cited.

\section{0}

\section{Introduction}

Australian Stock Exchange 50 index is a market capitalisation weighted index comprising of the top 50 Australian stocks. Price and market capitalisation weighted indexes are the most popular of all index methods and major stock indexes, for example, Dow Jones Industrial Average, S\&P500, FTSE100 and ASX50 are based on these methods. Both 
the market capitalisation and price weighted indexes are based on the concepts of modern portfolio theory and were seen to be the most efficient indexation methods. However, Arnott et al. $(2005 ; 2010 ; 2011)$ have challenged this idea by introducing the fundamental indexation methods that uses five fundamental factors, which are sales, book value, revenue, cash flow and employment. Empirical studies have shown that fundamental indexation has a value and small cap bias (Kaplan 2008, Hemminki et al. 2008, Hsu et al. 2006, Blitz et al. 2008, Estrada 2006, Chen et al. 2007, Siegel 2006, Mar et al. 2007 and Blitz et al. 2010). However, it is not clear if this indexation method clearly outperforms the market capitalisation or price weighted methods. Advantages of the price and market capitalisation methods is that they automatically rebalance themselves as the price of each stock within the index changes, though the disadvantage is that they are seen to overweight overvalued stocks and underweight undervalued stocks. On the other hand, fundamental indexes need to be rebalanced at regular intervals and seem to have a value and small cap stock bias compared to price and market cap weighted indexes.

Mar et al. (2009) analyzed fundamental indexation using ASX price data for the time period 1996 - 2005. However, they find that the fundamental indexation method performs better than the market cap weighted indexes mainly due to the value tilt in the fundamental indexation technique. They argue that the presence of a value tilt signifies that this method may not be a more efficient indexation method. Basu and Forbes (2013) state that based on an empirical study on five-year rolling basis Australian stocks show a greater out performance of the fundamental index compared to market cap weighted index even when considering the value, small cap bias and higher transaction costs.

Equal weighted and risk weighted index methods are the other alternative to the price, market cap and fundamental indexes. Equal weighted indexes provide the same weight to all stocks within the index regardless of their riskreturn profile. However, this method is seen as a 'take profit' approach as stocks that increase in value are sold to bring them back to equal weight and the opposite occurs with the stocks that fall in price. A disadvantage of the equal weighted index method is that it requires rebalancing at regular intervals and has a high tracking error to market capitalization indexes (Bernartzi and Thaler 2001, Windcliff and Boyle 2004 and DeMiguel et al., 2007).

While, equal weighted indexes have been used for equity indexation, risk weighted indexes are another index method that has been developed over the past few years. There are a few different types of risk weighted indexes that look at reducing risk through diversification. Risk weighted indexes that have been developed are minimum variance (MV), equally weighted portfolio, maximum Sharpe ratio (MSR), most diversified portfolio (MDP), equallyweighted risk distribution (ERC) portfolio and semi-variance portfolio (Demey et al. 2010). Risk weighted indexes like the risk cluster equal weighted index is similar to the equal weighted index except that the stocks are segregated into clusters based on their risk level, where each risk cluster has an equal weight in the index. An example of the risk cluster index is the QS Investors' diversification based index that weights risk clusters based on country and sector associations (Chow et al. 2011).

Other risk weighted indexes like minimum variance and mean variance optimization index methods intent to reduce or optimize the volatility of the portfolio respectively in line with modern portfolio theory. A major disadvantage of such methods is that they are hard to calculate due to the high dimensionality of the covariance matrix that is required before stock weights can be calculated for this index. Though, based on empirical studies, it has been seen that the minimum variance index outperforms the market capitalisation index in a falling market, but lags in a rising market (Haugen and Baker 1991; Clarke, de Silva, and Thorley 2006; Chia et al. 2011). On the other hand, Choueifaty and Coignard (2008) developed a risk weighted method that utilises the Sharpe ratio in order to find the weight of these stocks within the index. While, Amenc, Goltz,Martellini and Retkowsky (2010) develop a competing index method that weights stocks utilizing returns and semi-volatility. Their justification is that investors are only concerned about falling stock prices, as a result only negative returns and semi-variance should be considered when developing an index. Advantages of the risk weighted index methods is that they assign stock weights based on the risk-return trade off compared to an equal weighted index method that does not consider such factors. Also, these risk weighted indexes perform well in falling markets as they either have stocks that have lower variance or have higher diversification.

We have briefly discussed price, market Capitalisation, equal and risk weighted index methods and each of these methods have advantages and disadvantages. In general, price and market cap weighted indexes are seen to overweight overvalued stocks and are not seen as the optimal indexation methods. Fundamental indexes on the other hand are seen to be value and small cap biased and there is competing evidence if this index method is superior to the price and market cap weighted indexes. Fundamental indexes are also seen as an index method that generates a higher alpha due to the value and small cap bias. In comparison, risk weighted indexes concentrate on risk reduction through diversification. The intent of this paper is to introduce three new index methods called the risk weighted alpha, treynor's square index and appraisal ratio indexation, that identify stocks which have increasing returns and lower volatility using the ASX50 index. These methods re-weight stocks in the ASX50 index based on the risk weighted alpha, treynor's square or appraisal ratio of that stock. The next section provides an 
explanation of the risk weighted alpha index, treynor's square index and appraisal ratio index construction and then it provides examples on how these methods can be applied for index construction. It also shows that the risk weighted alpha method is a better method than the market cap and price weighted ASX50 index and the other two new methods (treynor's square and appraisal ratio ASX50 indexes).

\subsection{Risk weighted alpha index construction for the ASX50 index}

Risk Weighted Alpha (RWA) index method intends to provide higher weight to stocks that have higher returns and lower variance. In order to achieve this it uses Jensen's alpha to identify stocks that provide higher actual returns compared to their expected returns when considering systematic risk as derived by the capital asset pricing model. However, it is important that we find risk weighted Jensen's alpha, as some of these stocks may take on higher risk in order to provide a higher return. As a result, the risk weighted Jensen's alpha will identify stocks that provide the highest alpha per unit risk. The following formula explains the risk weighted alpha index method:

Where,

$$
\text { Risk Adjusted Jensen's Alpha }=\frac{R_{i}-\left[R_{f}+\beta_{i}\left(R_{m}-R_{f}\right)\right]}{\sigma_{i}}
$$

$$
\begin{gathered}
R_{i}=\text { actual return on the stock } \\
R_{f}=\text { risk free rate } \\
R_{m}=\text { return on the market index } \\
\beta_{i}=\text { beta of the stock } \\
\sigma_{i}=\text { standard deviation of the stock }
\end{gathered}
$$

Two alternate approaches would be to use Treynor's square and the Appraisal ratio to develop an index. Formula for Treynor's square is simply stated as Alpha/Beta for the stock. This measure intends to analyse risk adjusted performance and assumes that a stock with higher beta should also provide a higher alpha in order to cover for the higher systematic risk.

$$
\text { Treynor's Square }=\frac{\text { Alpha }}{\text { Beta }}
$$

In comparison, the Appraisal ratio intends to find the risk adjusted alpha is stated as alpha divided by non-market volatility. Where, non-market volatility is calculated as: square root of stock.

$$
\text { Appraisal Ratio }=\frac{\text { Alpha }}{\left.\sqrt{\left(\sigma_{i}^{2}\right.}-\beta_{i} \sigma_{m}^{2}\right)}
$$

Effectively, once the risk weighted alpha, treynor's square and appraisal ratio are obtained, this model assigns index weight to each stock based on this value. As it re-weights the ASX50 index, it is possible that some stocks have a negative risk weighted alpha. As a result, these stocks will have a negative index weight, which would mean that the investor should short these stocks as they do not provide sufficient return compared to the risk associated with them. It is also noticed that stocks with a positive risk weighted alpha over a significant period are those stocks that have consistently increasing stock prices over time with lower stock price volatility. It also compares the performance of the risk weighted alpha with the Treynor's square and Appraisal ratio. This paper uses the ASX50 index as an example in the next section in order to explain the risk weighted alpha index method. Financial data used in this paper was obtained from the Thomson Reuters Tick History database using daily price data for the ASX50 index from $2^{\text {nd }}$ January 2002 to $31^{\text {st }}$ December 2012.

\subsection{Performance characteristics - risk weighted alpha and ASX50 index}

ASX50 index is a market capitalization weighted index comprised of the top 50 stocks listed on the Australian Stock Exchange. This paper analyses if the risk weighted alpha, treynor's square or appraisal ratio method will provide superior return and lower variance by re-weighting the stocks in the ASX50 index. Performance of the risk weighted alpha against the market cap weighted ASX50, treynor's square and appraisal ratio indexes are provided below:

Table: 01 (A) Performance - Risk Weighted Alpha Index

\begin{tabular}{cccccccccccc}
\hline \multicolumn{1}{c}{ Index } & $\mathbf{2 0 0 3}$ & $\mathbf{2 0 0 4}$ & $\mathbf{2 0 0 5}$ & $\mathbf{2 0 0 6}$ & $\mathbf{2 0 0 7}$ & $\mathbf{2 0 0 8}$ & $\mathbf{2 0 0 9}$ & $\mathbf{2 0 1 0}$ & $\mathbf{2 0 1 1}$ & $\mathbf{2 0 1 2}$ & Index Return \\
\hline \hline ASX50 Index & $9.78 \%$ & $20.74 \%$ & $16.70 \%$ & $18.32 \%$ & $12.63 \%$ & $-47.41 \%$ & $29.07 \%$ & $-1.37 \%$ & $-13.78 \%$ & $14.34 \%$ & $59.01 \%$ \\
\hline Risk Weighted Alpha ASX50 Index & $51.80 \%$ & $47.55 \%$ & $39.66 \%$ & $16.69 \%$ & $28.03 \%$ & $6.60 \%$ & $26.02 \%$ & $21.14 \%$ & $0.38 \%$ & $8.33 \%$ & $246.20 \%$ \\
\hline
\end{tabular}


Table: 01 (B) Performance - Treynor's Square Index

\begin{tabular}{|c|c|c|c|c|c|c|c|c|c|c|c|}
\hline Index & 2003 & 2004 & 2005 & 2006 & 2007 & 2008 & 2009 & 2010 & 2011 & 2012 & Index Return \\
\hline ASX50 Index & $9.78 \%$ & $20.74 \%$ & $16.70 \%$ & $18.32 \%$ & $12.63 \%$ & $-47.41 \%$ & $29.07 \%$ & $-1.37 \%$ & $-13.78 \%$ & $14.34 \%$ & $59.01 \%$ \\
\hline Treynor's Square ASX50 Index & $49.01 \%$ & $35.33 \%$ & $25.45 \%$ & $25.23 \%$ & $0.92 \%$ & $-33.25 \%$ & $50.16 \%$ & $24.93 \%$ & $61.09 \%$ & $16.22 \%$ & $255.09 \%$ \\
\hline
\end{tabular}

Table: 01 (C) Performance - Appraisal Ratio Index

\begin{tabular}{lccccccccccc}
\hline \multicolumn{1}{c}{ Index } & $\mathbf{2 0 0 3}$ & $\mathbf{2 0 0 4}$ & $\mathbf{2 0 0 5}$ & $\mathbf{2 0 0 6}$ & $\mathbf{2 0 0 7}$ & $\mathbf{2 0 0 8}$ & $\mathbf{2 0 0 9}$ & $\mathbf{2 0 1 0}$ & $\mathbf{2 0 1 1}$ & $\mathbf{2 0 1 2}$ & Index Return \\
\hline \hline ASX50 Index & $9.78 \%$ & $20.74 \%$ & $16.70 \%$ & $18.32 \%$ & $12.63 \%$ & $-47.41 \%$ & $29.07 \%$ & $-1.37 \%$ & $-13.78 \%$ & $14.34 \%$ & $59.01 \%$ \\
\hline Appraisal Ratio ASX50 Index & $46.05 \%$ & $44.59 \%$ & $36.57 \%$ & $15.60 \%$ & $26.89 \%$ & $5.40 \%$ & $26.51 \%$ & $18.26 \%$ & $1.61 \%$ & $11.13 \%$ & $232.62 \%$ \\
\hline
\end{tabular}

We notice that the index return for the risk weighted alpha (246.20\%) and treynor's square index (255.09\%) methods is higher than the market cap weighted ASX50 and the appraisal ratio based method (232.62\%). The reason for the higher returns is that these methods weight stocks with higher alphas per unit risk. If stocks consistently provide higher alpha per unit risk over an extended period of time, then these methods provide a higher weight to that stock in the index. Existing methods do not specifically weight stocks based on their alpha. Though, only high performing stocks will consistently be able to provide a positive alpha per unit risk over an extended period of time. If a stock however does provide a higher alpha, but it also has a higher level of volatility in its returns. Then, it is unlikely that this stock will be provided an above average weight due to the higher volatility.

Let's look at the profiles of the best and worst performing stocks in each of these methods (see graphs below). Using the risk weighted alpha index method; data shows that the Oil Search stock performed well as it had a higher risk weighted alpha. If you notice the graph below, the stock price has steadily increased from 2002 to 2013. On the contrary, the worst performing stock in the risk weighted alpha index was Qantas, and the graph below shows that the stock price had high volatility over the sample period (2002-2012) and the stock price is less than half of what it was in January 2002.

Given the figure panel A, Now, let's compare the risk weighted alpha graphs with those provided by the treynor's square. Santos, Newcrest mining, Origin energy and Iluka resources are some of the stocks that have a high weight in the treynor's square index. On the other hand, if you notice that Newcrest and Iluka have sharply decreasing returns, however they still have a high weight in this index. The reason for this higher weight as it averages the alpha against the beta for each stock over the sample period (2002-2012), which means that this method does not pick stocks with increasing returns and lower volatility. Instead, it picks stocks that will provide the highest alpha and if the portfolio is sufficiently diversified as in the case of the ASX50 index. Then, this portfolio will provide increasing returns as the unsystematic risk gets mostly diversified. In comparison, you can see the Intec Pivot stock price graph below, as this stock has a lower weight in the treynor's square index - primarily as it's average alpha compared to its systematic risk is lower than the other stocks in the ASX50 index. 


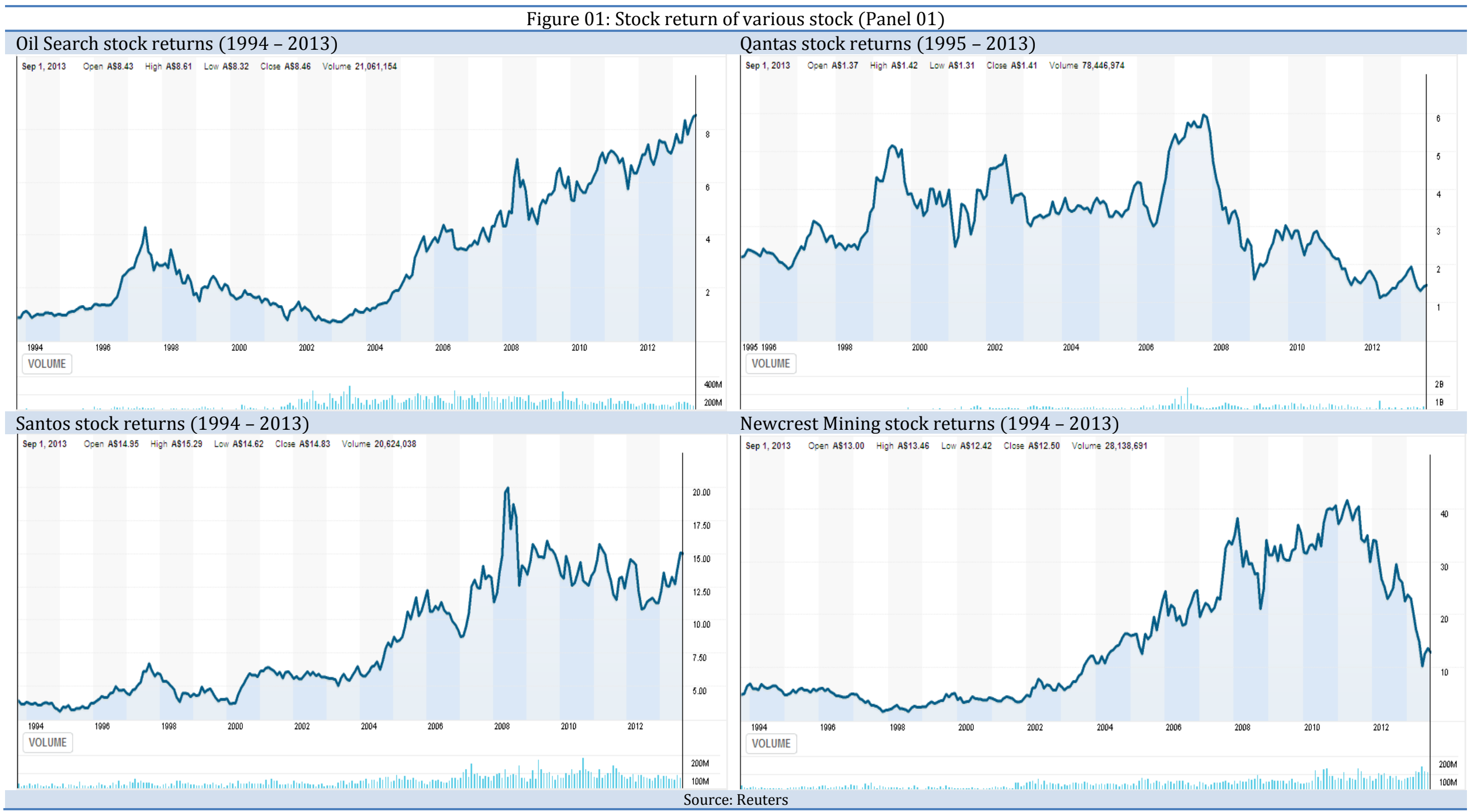




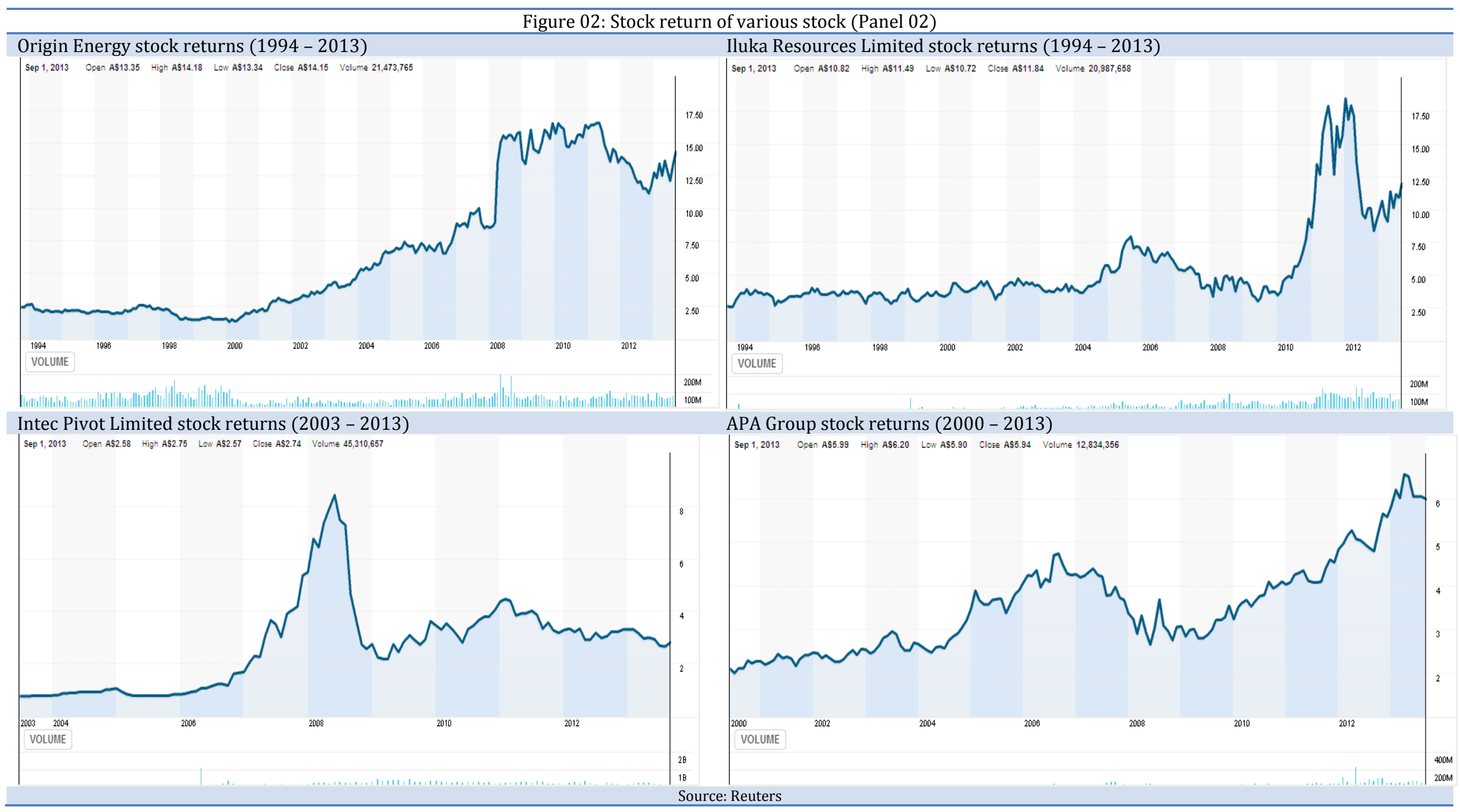




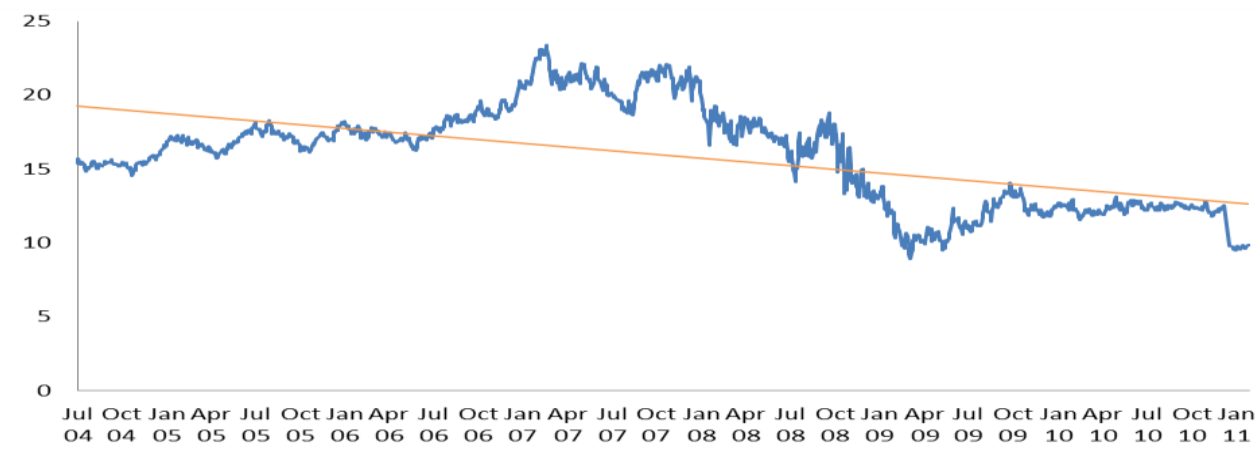

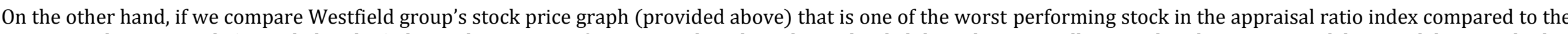

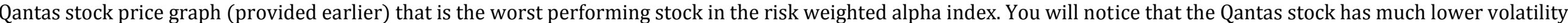

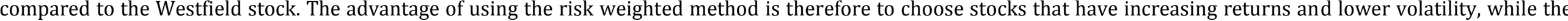

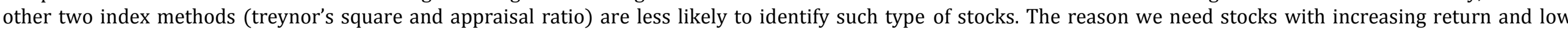
volatility is to reduce the need to rebalance a passive index more frequently. The risk-return characteristics of these indexes is provided below.

Table 02: Risk Weighted Alpha Index - Risk/Return Characteristics (January 2002 - December 2012)

\begin{tabular}{|c|c|c|c|c|c|c|c|}
\hline Stock Name & $\begin{array}{l}\text { Standard } \\
\text { Deviation } \\
\end{array}$ & Beta & $\begin{array}{c}\text { Expected } \\
\text { Return } \\
\end{array}$ & Alpha & $\begin{array}{c}\text { Risk Adjusted } \\
\text { Alpha }\end{array}$ & $\begin{array}{c}\text { RWA Index } \\
\text { Weight } \\
\end{array}$ & $\begin{array}{c}\text { Absolute RWA Index } \\
\text { Weight }\end{array}$ \\
\hline OIL SEARCH & 0.2838 & 0.4058 & $27.48 \%$ & 2.8822 & 10.1545 & $10.4498 \%$ & $17.6100 \%$ \\
\hline CC AMATIL & 0.1214 & 0.2230 & $17.79 \%$ & 1.0351 & 8.5280 & $8.7761 \%$ & $14.7894 \%$ \\
\hline APA GROUP & 0.1214 & 0.2537 & $19.41 \%$ & 0.9385 & 7.7301 & $7.9549 \%$ & $13.4056 \%$ \\
\hline FORTESCUE & 1.0754 & 2.6188 & $144.91 \%$ & 7.2548 & 6.7462 & $6.9424 \%$ & $11.6993 \%$ \\
\hline ORIGIN ENE & 0.2572 & -0.4572 & $-18.31 \%$ & 1.7048 & 6.6291 & $6.8219 \%$ & $11.4963 \%$ \\
\hline NEWCREST & 0.2747 & 0.1308 & $12.89 \%$ & 1.7866 & 6.5035 & $6.6926 \%$ & $11.2784 \%$ \\
\hline BHP BLT & 0.2345 & 0.6782 & $41.94 \%$ & 1.3990 & 5.9666 & $6.1401 \%$ & $10.3473 \%$ \\
\hline SANTOS & 0.2059 & -0.0765 & $1.89 \%$ & 1.1704 & 5.6838 & $5.8491 \%$ & $9.8568 \%$ \\
\hline CSHARE & 0.2991 & 0.8489 & $51.00 \%$ & 1.6268 & 5.4399 & $5.5981 \%$ & $9.4339 \%$ \\
\hline CSL & 0.2810 & 0.4393 & $29.26 \%$ & 1.4688 & 5.2268 & $5.3788 \%$ & $9.0643 \%$ \\
\hline WOOLWORTHS & 0.1794 & 0.5247 & $33.79 \%$ & 0.8010 & 4.4646 & $4.5944 \%$ & $7.7425 \%$ \\
\hline ILUKA RES & 0.4374 & -0.6351 & $-27.75 \%$ & 1.7855 & 4.0820 & $4.2007 \%$ & $7.0790 \%$ \\
\hline WOODSIDE & 0.2898 & 0.7698 & $46.80 \%$ & 1.0137 & 3.4982 & $3.6000 \%$ & $6.0667 \%$ \\
\hline TRANSURBAN & 0.1555 & 0.3294 & $23.43 \%$ & 0.5433 & 3.4948 & $3.5964 \%$ & $6.0606 \%$ \\
\hline
\end{tabular}




\begin{tabular}{|c|c|c|c|c|c|c|c|}
\hline SONIC HLTH & 0.2140 & 0.4676 & $30.76 \%$ & 0.7394 & 3.4545 & $3.5550 \%$ & $5.9908 \%$ \\
\hline WORLEYPARS & 0.6281 & 2.2503 & $125.36 \%$ & 2.1527 & 3.4270 & $3.5267 \%$ & $-5.9432 \%$ \\
\hline ASX & 0.3039 & 0.9302 & $55.31 \%$ & 0.8211 & 2.7018 & $2.7804 \%$ & $-4.6855 \%$ \\
\hline BRAMB LTD & 0.2078 & 0.5955 & $37.55 \%$ & 0.5484 & 2.6396 & $2.7164 \%$ & $-4.5776 \%$ \\
\hline AGL ENERGY & 0.0972 & -0.2359 & $-6.57 \%$ & 0.2494 & 2.5642 & $2.6388 \%$ & $-4.4468 \%$ \\
\hline CFS RETAIL & 0.1145 & 0.3283 & $23.37 \%$ & 0.2791 & 2.4382 & $2.5091 \%$ & $-4.2284 \%$ \\
\hline ASCIANO & 0.7176 & 0.0867 & $10.55 \%$ & 1.5983 & 2.2273 & $2.2920 \%$ & $-3.8625 \%$ \\
\hline INSUR.AUST & 0.3043 & 0.3131 & $22.57 \%$ & 0.6752 & 2.2192 & $2.2837 \%$ & $-3.8485 \%$ \\
\hline GPT & 1.2576 & 0.8885 & $53.10 \%$ & 2.6685 & 2.1220 & $2.1837 \%$ & $-3.6799 \%$ \\
\hline WESTPAC & 0.2254 & 0.8434 & $50.70 \%$ & 0.4612 & 2.0460 & $2.1055 \%$ & $-3.5481 \%$ \\
\hline ORICA & 0.3695 & 1.3467 & $77.41 \%$ & 0.5524 & 1.4950 & $1.5384 \%$ & $-2.5925 \%$ \\
\hline WESTFTRUST & 0.0683 & 0.0407 & $8.11 \%$ & 0.0953 & 1.3950 & $1.4356 \%$ & $-2.4192 \%$ \\
\hline CWLTH BANK & 0.3226 & 1.1845 & $68.80 \%$ & 0.4294 & 1.3314 & $1.3701 \%$ & $-2.3089 \%$ \\
\hline AMCOR & 0.0890 & 0.1282 & $12.75 \%$ & 0.1172 & 1.3166 & $1.3549 \%$ & $-2.2833 \%$ \\
\hline QBE INSUR. & 0.2764 & 0.6579 & $40.86 \%$ & 0.2843 & 1.0288 & $1.0587 \%$ & $-1.7841 \%$ \\
\hline MACQ GROUP & 0.4041 & 1.4573 & $83.28 \%$ & 0.3989 & 0.9872 & $1.0159 \%$ & $-1.7120 \%$ \\
\hline AURIZON & 0.0312 & 0.0169 & $6.84 \%$ & 0.0302 & 0.9688 & $0.9970 \%$ & $-1.6801 \%$ \\
\hline ANZ BANK & 0.2333 & 0.8776 & $52.52 \%$ & 0.2036 & 0.8726 & $0.8979 \%$ & $-1.5132 \%$ \\
\hline RIO TINTO & 0.5096 & 1.8064 & $101.80 \%$ & 0.4328 & 0.8493 & $0.8740 \%$ & $-1.4729 \%$ \\
\hline TELSTRA & 0.1678 & 0.1350 & $13.11 \%$ & 0.0738 & 0.4400 & $0.4528 \%$ & $-0.7630 \%$ \\
\hline 21STCENFOX & 0.0000 & 0.0000 & $5.95 \%$ & -0.0595 & 0.0000 & $0.0000 \%$ & $0.0000 \%$ \\
\hline WESFARMER & 0.3386 & 1.2064 & $69.96 \%$ & -0.0058 & -0.0172 & $-0.0177 \%$ & $0.0298 \%$ \\
\hline INCITEC PV & 0.7450 & 2.4273 & $134.75 \%$ & -0.0221 & -0.0297 & $-0.0306 \%$ & $0.0515 \%$ \\
\hline SUNCORP & 0.2400 & 0.8095 & $48.90 \%$ & -0.0758 & -0.3159 & $-0.3251 \%$ & $0.5479 \%$ \\
\hline TOLLHOLDGS & 0.3526 & 1.1436 & $66.63 \%$ & -0.3388 & -0.9608 & $-0.9888 \%$ & $1.6663 \%$ \\
\hline LEND LEASE & 0.3378 & 1.3167 & $75.82 \%$ & -0.3470 & -1.0274 & $-1.0573 \%$ & $1.7818 \%$ \\
\hline DEXUS PROP & 0.2647 & 0.9278 & $55.18 \%$ & -0.2751 & -1.0393 & $-1.0695 \%$ & $1.8023 \%$ \\
\hline STOCKLAND. & 0.2189 & 0.8522 & $51.17 \%$ & -0.2696 & -1.2320 & $-1.2678 \%$ & $2.1365 \%$ \\
\hline SYDAIRPORT & 0.1330 & 0.1654 & $14.72 \%$ & -0.1919 & -1.4430 & $-1.4850 \%$ & $2.5025 \%$ \\
\hline NAT. BANK & 0.2081 & 0.7092 & $43.58 \%$ & -0.3012 & -1.4470 & $-1.4891 \%$ & $2.5095 \%$ \\
\hline WESTFIELDG & 0.1951 & 0.6382 & $39.81 \%$ & -0.3976 & -2.0373 & $-2.0966 \%$ & $3.5331 \%$ \\
\hline
\end{tabular}




\begin{tabular}{|c|c|c|c|c|c|c|c|}
\hline CROWN & 0.2807 & 0.9554 & $56.64 \%$ & -0.5838 & -2.0795 & $-2.1400 \%$ & $3.6063 \%$ \\
\hline AMP & 0.3436 & 0.9749 & $57.68 \%$ & -0.8422 & -2.4508 & $-2.5221 \%$ & $4.2502 \%$ \\
\hline MIRVAC GRP & 0.4625 & 1.7058 & $96.46 \%$ & -1.1983 & -2.5907 & $-2.6661 \%$ & $4.4928 \%$ \\
\hline GOOD GROUP & 0.5712 & 2.0151 & $112.87 \%$ & -1.8577 & -3.2522 & $-3.3468 \%$ & $5.6400 \%$ \\
\hline QANTAS & 0.2990 & 1.1307 & $65.95 \%$ & -1.0690 & -3.5757 & $-3.6797 \%$ & $6.2010 \%$ \\
\hline Total & 0.2437 & 0.8548 & $55.10 \%$ & 0.1386 & 43.3260 & $100.0000 \%$ & $100.0000 \%$ \\
\hline
\end{tabular}

Table 03: Treynor's Square Index - Risk/Return Characteristics (January 2002 - December 2012)

\begin{tabular}{|c|c|c|c|c|c|c|c|}
\hline Stock Name & $\begin{array}{l}\text { Standard } \\
\text { Deviation } \\
\end{array}$ & Beta & $\begin{array}{c}\text { Expected } \\
\text { Return } \\
\end{array}$ & Alpha & Treynor's Square & $\begin{array}{c}\text { TS Index } \\
\text { Weight } \\
\end{array}$ & Absolute TS Index Weight \\
\hline ASCIANO & 0.7176 & 0.0867 & 0.1055 & 1.5983 & 18.4297 & $36.7574 \%$ & $17.6685 \%$ \\
\hline SANTOS & 0.2059 & -0.0765 & $1.89 \%$ & 1.1704 & -15.2975 & $-30.5103 \%$ & $14.6657 \%$ \\
\hline NEWCREST & 0.2747 & 0.1308 & $12.89 \%$ & 1.7866 & 13.6625 & $27.2495 \%$ & $13.0983 \%$ \\
\hline OIL SEARCH & 0.2838 & 0.4058 & $27.48 \%$ & 2.8822 & 7.1026 & $14.1659 \%$ & $6.8093 \%$ \\
\hline CC AMATIL & 0.1214 & 0.2230 & $17.79 \%$ & 1.0351 & 4.6410 & $9.2564 \%$ & $4.4494 \%$ \\
\hline ORIGIN ENE & 0.2572 & -0.4572 & $-18.31 \%$ & 1.7048 & -3.7286 & $-7.4366 \%$ & $3.5746 \%$ \\
\hline APA GROUP & 0.1214 & 0.2537 & $19.41 \%$ & 0.9385 & 3.6998 & $7.3791 \%$ & $3.5470 \%$ \\
\hline CSL & 0.2810 & 0.4393 & $29.26 \%$ & 1.4688 & 3.3432 & $6.6680 \%$ & $3.2052 \%$ \\
\hline GPT & 1.2576 & 0.8885 & $53.10 \%$ & 2.6685 & 3.0034 & $5.9902 \%$ & $2.8794 \%$ \\
\hline ILUKA RES & 0.4374 & -0.6351 & $-27.75 \%$ & 1.7855 & -2.8112 & $-5.6068 \%$ & $2.6951 \%$ \\
\hline FORTESCUE & 1.0754 & 2.6188 & $144.91 \%$ & 7.2548 & 2.7703 & $5.5252 \%$ & $2.6559 \%$ \\
\hline WESTFTRUST & 0.0683 & 0.0407 & $8.11 \%$ & 0.0953 & 2.3423 & $4.6716 \%$ & $2.2455 \%$ \\
\hline INSUR.AUST & 0.3043 & 0.3131 & $22.57 \%$ & 0.6752 & 2.1562 & $4.3005 \%$ & $2.0672 \%$ \\
\hline BHP BLT & 0.2345 & 0.6782 & $41.94 \%$ & 1.3990 & 2.0628 & $4.1142 \%$ & $1.9776 \%$ \\
\hline CSHARE & 0.2991 & 0.8489 & $51.00 \%$ & 1.6268 & 1.9164 & $3.8221 \%$ & $1.8372 \%$ \\
\hline AURIZON & 0.0312 & 0.0169 & $6.84 \%$ & 0.0302 & 1.7925 & $3.5751 \%$ & $1.7185 \%$ \\
\hline TRANSURBAN & 0.1555 & 0.3294 & $23.43 \%$ & 0.5433 & 1.6495 & $3.2899 \%$ & $1.5814 \%$ \\
\hline SONIC HLTH & 0.2140 & 0.4676 & $30.76 \%$ & 0.7394 & 1.5811 & $3.1535 \%$ & $1.5158 \%$ \\
\hline WOOLWORTHS & 0.1794 & 0.5247 & $33.79 \%$ & 0.8010 & 1.5266 & $3.0448 \%$ & $1.4636 \%$ \\
\hline WOODSIDE & 0.2898 & 0.7698 & $46.80 \%$ & 1.0137 & 1.3168 & $2.6263 \%$ & $1.2624 \%$ \\
\hline SYDAIRPORT & 0.1330 & 0.1654 & $14.72 \%$ & -0.1919 & -1.1603 & $-2.3142 \%$ & $1.1124 \%$ \\
\hline
\end{tabular}




\begin{tabular}{|c|c|c|c|c|c|c|c|}
\hline AGL ENERGY & 0.0972 & -0.2359 & $-6.57 \%$ & 0.2494 & -1.0571 & $-2.1084 \%$ & $1.0135 \%$ \\
\hline WORLEYPARS & 0.6281 & 2.2503 & $125.36 \%$ & 2.1527 & 0.9566 & $1.9079 \%$ & $0.9171 \%$ \\
\hline QANTAS & 0.2990 & 1.1307 & $65.95 \%$ & -1.0690 & -0.9455 & $-1.8857 \%$ & $0.9064 \%$ \\
\hline GOOD GROUP & 0.5712 & 2.0151 & $112.87 \%$ & -1.8577 & -0.9219 & $-1.8387 \%$ & $0.8838 \%$ \\
\hline BRAMB LTD & 0.2078 & 0.5955 & $37.55 \%$ & 0.5484 & 0.9209 & $1.8367 \%$ & $0.8828 \%$ \\
\hline AMCOR & 0.0890 & 0.1282 & $12.75 \%$ & 0.1172 & 0.9146 & $1.8241 \%$ & $0.8768 \%$ \\
\hline ASX & 0.3039 & 0.9302 & $55.31 \%$ & 0.8211 & 0.8827 & $1.7606 \%$ & $0.8463 \%$ \\
\hline AMP & 0.3436 & 0.9749 & $57.68 \%$ & -0.8422 & -0.8639 & $-1.7230 \%$ & $0.8282 \%$ \\
\hline CFS RETAIL & 0.1145 & 0.3283 & $23.37 \%$ & 0.2791 & 0.8503 & $1.6959 \%$ & $0.8152 \%$ \\
\hline MIRVAC GRP & 0.4625 & 1.7058 & $96.46 \%$ & -1.1983 & -0.7025 & $-1.4010 \%$ & $0.6735 \%$ \\
\hline WESTFIELDG & 0.1951 & 0.6382 & $39.81 \%$ & -0.3976 & -0.6230 & $-1.2425 \%$ & $0.5972 \%$ \\
\hline CROWN & 0.2807 & 0.9554 & $56.64 \%$ & -0.5838 & -0.6110 & $-1.2187 \%$ & $0.5858 \%$ \\
\hline TELSTRA & 0.1678 & 0.1350 & $13.11 \%$ & 0.0738 & 0.5470 & $1.0910 \%$ & $0.5244 \%$ \\
\hline WESTPAC & 0.2254 & 0.8434 & $50.70 \%$ & 0.4612 & 0.5469 & $1.0908 \%$ & $0.5243 \%$ \\
\hline QBE INSUR. & 0.2764 & 0.6579 & $40.86 \%$ & 0.2843 & 0.4322 & $0.8620 \%$ & $0.4144 \%$ \\
\hline NAT. BANK & 0.2081 & 0.7092 & $43.58 \%$ & -0.3012 & -0.4247 & $-0.8470 \%$ & $0.4071 \%$ \\
\hline ORICA & 0.3695 & 1.3467 & $77.41 \%$ & 0.5524 & 0.4102 & $0.8182 \%$ & $0.3933 \%$ \\
\hline CWLTH BANK & 0.3226 & 1.1845 & $68.80 \%$ & 0.4294 & 0.3626 & $0.7231 \%$ & $0.3476 \%$ \\
\hline STOCKLAND. & 0.2189 & 0.8522 & $51.17 \%$ & -0.2696 & -0.3164 & $-0.6311 \%$ & $0.3033 \%$ \\
\hline DEXUS PROP & 0.2647 & 0.9278 & $55.18 \%$ & -0.2751 & -0.2965 & $-0.5915 \%$ & $0.2843 \%$ \\
\hline TOLLHOLDGS & 0.3526 & 1.1436 & $66.63 \%$ & -0.3388 & -0.2962 & $-0.5908 \%$ & $0.2840 \%$ \\
\hline MACQ GROUP & 0.4041 & 1.4573 & $83.28 \%$ & 0.3989 & 0.2737 & $0.5460 \%$ & $0.2624 \%$ \\
\hline LEND LEASE & 0.3378 & 1.3167 & $75.82 \%$ & -0.3470 & -0.2636 & $-0.5257 \%$ & $0.2527 \%$ \\
\hline RIO TINTO & 0.5096 & 1.8064 & $101.80 \%$ & 0.4328 & 0.2396 & $0.4779 \%$ & $0.2297 \%$ \\
\hline ANZ BANK & 0.2333 & 0.8776 & $52.52 \%$ & 0.2036 & 0.2320 & $0.4627 \%$ & $0.2224 \%$ \\
\hline SUNCORP & 0.2400 & 0.8095 & $48.90 \%$ & -0.0758 & -0.0937 & $-0.1868 \%$ & $0.0898 \%$ \\
\hline INCITEC PV & 0.7450 & 2.4273 & $134.75 \%$ & -0.0221 & -0.0091 & $-0.0182 \%$ & $0.0087 \%$ \\
\hline WESFARMER & 0.3386 & 1.2064 & $69.96 \%$ & -0.0058 & -0.0048 & $-0.0096 \%$ & $0.0046 \%$ \\
\hline 21STCENFOX & 0.0000 & 0.0000 & $5.95 \%$ & -0.0595 & 0.0000 & $0.0000 \%$ & $0.0000 \%$ \\
\hline Total & 0.2437 & 0.8548 & $55.10 \%$ & 0.1386 & 43.3260 & $100.0000 \%$ & $100.0000 \%$ \\
\hline
\end{tabular}


Table 04: Appraisal Ratio Index - Risk/Return Characteristics (January 2002 - December 2012)

\begin{tabular}{|c|c|c|c|c|c|c|c|}
\hline Stock Name & $\begin{array}{l}\text { Standard } \\
\text { Deviation } \\
\end{array}$ & Beta & $\begin{array}{c}\text { Expected } \\
\text { Return } \\
\end{array}$ & Alpha & Appraisal Ratio & $\begin{array}{c}\text { AR Index } \\
\text { Weight } \\
\end{array}$ & $\begin{array}{c}\text { Absolute AR Index } \\
\text { Weight } \\
\end{array}$ \\
\hline APA GROUP & 0.1214 & 0.2537 & $19.41 \%$ & 0.9385 & 19.9135 & $15.1503 \%$ & $22.6420 \%$ \\
\hline CC AMATIL & 0.1214 & 0.2230 & $17.79 \%$ & 1.0351 & 16.9597 & $12.9031 \%$ & $19.2835 \%$ \\
\hline OIL SEARCH & 0.2838 & 0.4058 & $27.48 \%$ & 2.8822 & 11.7146 & $8.9125 \%$ & $13.3197 \%$ \\
\hline WOOLWORTHS & 0.1794 & 0.5247 & $33.79 \%$ & 0.8010 & 10.0957 & $7.6809 \%$ & $11.4790 \%$ \\
\hline BHP BLT & 0.2345 & 0.6782 & $41.94 \%$ & 1.3990 & 9.5403 & $7.2583 \%$ & $10.8475 \%$ \\
\hline CSHARE & 0.2991 & 0.8489 & $51.00 \%$ & 1.6268 & 7.4617 & $5.6769 \%$ & $8.4841 \%$ \\
\hline FORTESCUE & 1.0754 & 2.6188 & $144.91 \%$ & 7.2548 & 7.1580 & $5.4459 \%$ & $8.1388 \%$ \\
\hline NEWCREST & 0.2747 & 0.1308 & $12.89 \%$ & 1.7866 & 6.8008 & $5.1741 \%$ & $7.7326 \%$ \\
\hline CSL & 0.2810 & 0.4393 & $29.26 \%$ & 1.4688 & 6.1368 & $4.6689 \%$ & $6.9776 \%$ \\
\hline TRANSURBAN & 0.1555 & 0.3294 & $23.43 \%$ & 0.5433 & 6.1074 & $4.6466 \%$ & $6.9443 \%$ \\
\hline ORIGIN ENE & 0.2572 & -0.4572 & $-18.31 \%$ & 1.7048 & 5.7241 & $4.3549 \%$ & $6.5084 \%$ \\
\hline SANTOS & 0.2059 & -0.0765 & $1.89 \%$ & 1.1704 & 5.4464 & $4.1437 \%$ & $6.1927 \%$ \\
\hline SONIC HLTH & 0.2140 & 0.4676 & $30.76 \%$ & 0.7394 & 4.9040 & $3.7310 \%$ & $5.5760 \%$ \\
\hline WESTPAC & 0.2254 & 0.8434 & $50.70 \%$ & 0.4612 & 4.8094 & $3.6590 \%$ & $5.4683 \%$ \\
\hline WOODSIDE & 0.2898 & 0.7698 & $46.80 \%$ & 1.0137 & 4.7278 & $3.5969 \%$ & $5.3756 \%$ \\
\hline BRAMB LTD & 0.2078 & 0.5955 & $37.55 \%$ & 0.5484 & 4.6731 & $3.5553 \%$ & $-5.3134 \%$ \\
\hline WORLEYPARS & 0.6281 & 2.2503 & $125.36 \%$ & 2.1527 & 4.0430 & $3.0759 \%$ & $-4.5969 \%$ \\
\hline ASX & 0.3039 & 0.9302 & $55.31 \%$ & 0.8211 & 3.8097 & $2.8985 \%$ & $-4.3317 \%$ \\
\hline ILUKA RES & 0.4374 & -0.6351 & $-27.75 \%$ & 1.7855 & 3.7838 & $2.8787 \%$ & $-4.3022 \%$ \\
\hline AMCOR & 0.0890 & 0.1282 & $12.75 \%$ & 0.1172 & 2.9286 & $2.2281 \%$ & $-3.3299 \%$ \\
\hline AURIZON & 0.0312 & 0.0169 & $6.84 \%$ & 0.0302 & 2.5425 & $1.9344 \%$ & $-2.8909 \%$ \\
\hline INSUR.AUST & 0.3043 & 0.3131 & $22.57 \%$ & 0.6752 & 2.4314 & $1.8498 \%$ & $-2.7646 \%$ \\
\hline ASCIANO & 0.7176 & 0.0867 & $10.55 \%$ & 1.5983 & 2.2366 & $1.7016 \%$ & $-2.5430 \%$ \\
\hline GPT & 1.2576 & 0.8885 & $53.10 \%$ & 2.6685 & 2.1520 & $1.6373 \%$ & $-2.4469 \%$ \\
\hline ORICA & 0.3695 & 1.3467 & $77.41 \%$ & 0.5524 & 2.0867 & $1.5875 \%$ & $-2.3726 \%$ \\
\hline CWLTH BANK & 0.3226 & 1.1845 & $68.80 \%$ & 0.4294 & 2.0115 & $1.5303 \%$ & $-2.2871 \%$ \\
\hline ANZ BANK & 0.2333 & 0.8776 & $52.52 \%$ & 0.2036 & 1.9306 & $1.4688 \%$ & $-2.1952 \%$ \\
\hline WESTFTRUST & 0.0683 & 0.0407 & $8.11 \%$ & 0.0953 & 1.8480 & $1.4060 \%$ & $-2.1012 \%$ \\
\hline
\end{tabular}




\begin{tabular}{|c|c|c|c|c|c|c|c|}
\hline AGL ENERGY & 0.0972 & -0.2359 & $-6.57 \%$ & 0.2494 & 1.7167 & $1.3061 \%$ & $-1.9519 \%$ \\
\hline QBE INSUR. & 0.2764 & 0.6579 & $40.86 \%$ & 0.2843 & 1.3568 & $1.0323 \%$ & $-1.5427 \%$ \\
\hline MACQ GROUP & 0.4041 & 1.4573 & $83.28 \%$ & 0.3989 & 1.3198 & $1.0041 \%$ & $-1.5006 \%$ \\
\hline RIO TINTO & 0.5096 & 1.8064 & $101.80 \%$ & 0.4328 & 1.0481 & $0.7974 \%$ & $-1.1917 \%$ \\
\hline TELSTRA & 0.1678 & 0.1350 & $13.11 \%$ & 0.0738 & 0.5036 & $0.3831 \%$ & $-0.5726 \%$ \\
\hline CFS RETAIL & 0.1145 & 0.3283 & $23.37 \%$ & 0.2791 & 0.0000 & $0.0000 \%$ & $0.0000 \%$ \\
\hline 21STCENFOX & 0.0000 & 0.0000 & $5.95 \%$ & -0.0595 & 0.0000 & $0.0000 \%$ & $0.0000 \%$ \\
\hline WESFARMER & 0.3386 & 1.2064 & $69.96 \%$ & -0.0058 & -0.0247 & $-0.0188 \%$ & $0.0281 \%$ \\
\hline INCITEC PV & 0.7450 & 2.4273 & $134.75 \%$ & -0.0221 & -0.0336 & $-0.0255 \%$ & $0.0382 \%$ \\
\hline SUNCORP & 0.2400 & 0.8095 & $48.90 \%$ & -0.0758 & -0.5707 & $-0.4342 \%$ & $0.6489 \%$ \\
\hline TOLLHOLDGS & 0.3526 & 1.1436 & $66.63 \%$ & -0.3388 & -1.3004 & $-0.9894 \%$ & $1.4786 \%$ \\
\hline LEND LEASE & 0.3378 & 1.3167 & $75.82 \%$ & -0.3470 & -1.5661 & $-1.1915 \%$ & $1.7807 \%$ \\
\hline DEXUS PROP & 0.2647 & 0.9278 & $55.18 \%$ & -0.2751 & -1.7651 & $-1.3429 \%$ & $2.0069 \%$ \\
\hline SYDAIRPORT & 0.1330 & 0.1654 & $14.72 \%$ & -0.1919 & -1.9666 & $-1.4962 \%$ & $2.2361 \%$ \\
\hline AMP & 0.3436 & 0.9749 & $57.68 \%$ & -0.8422 & -3.1839 & $-2.4223 \%$ & $3.6202 \%$ \\
\hline CROWN & 0.2807 & 0.9554 & $56.64 \%$ & -0.5838 & -3.2813 & $-2.4964 \%$ & $3.7309 \%$ \\
\hline NAT. BANK & 0.2081 & 0.7092 & $43.58 \%$ & -0.3012 & -3.3020 & $-2.5121 \%$ & $3.7544 \%$ \\
\hline MIRVAC GRP & 0.4625 & 1.7058 & $96.46 \%$ & -1.1983 & -3.3267 & $-2.5310 \%$ & $3.7826 \%$ \\
\hline STOCKLAND. & 0.2189 & 0.8522 & $51.17 \%$ & -0.2696 & -3.5276 & $-2.6838 \%$ & $4.0109 \%$ \\
\hline GOOD GROUP & 0.5712 & 2.0151 & $112.87 \%$ & -1.8577 & -3.9006 & $-2.9676 \%$ & $4.4350 \%$ \\
\hline WESTFIELDG & 0.1951 & 0.6382 & $39.81 \%$ & -0.3976 & -4.8999 & $-3.7279 \%$ & $5.5713 \%$ \\
\hline QANTAS & 0.2990 & 1.1307 & $65.95 \%$ & -1.0690 & -5.8338 & $-4.4384 \%$ & $6.6331 \%$ \\
\hline Total & 0.2437 & 0.8548 & $55.10 \%$ & 0.1386 & 43.3260 & $100.0000 \%$ & $100.0000 \%$ \\
\hline
\end{tabular}


So, why does the risk weighted alpha index perform better? It intends to weight stocks that have stable and increasing returns with lower volatility, which is ideal for a passive index. Based on the risk weighted alpha (see table above), some of the stocks with negative risk weighted alphas should not be part of the ASX50 index for this sample period as they have substantially underperformed.

Ideally, an index should have stocks that represent an efficient portfolio, which means that other portfolios should not have a higher return or lower risk than them. As a result, it seems that the price or market cap weighted index may not perform as well as the risk weighted alpha index within this sample period $\left(2^{\text {nd }}\right.$ January $2002-31^{\text {st }}$ December 2012), as the risk weighted alpha index has lower risk and higher return than the ASX50 index. Effectively, the risk weighted alpha index should only have long positions, as these stocks would have the highest risk weighted alphas. However, as this paper was comparing the ASX50 index weights with the risk weighted alpha index method, based on the risk weighted alphas it seemed that some stocks did not provide sufficient return in relation to their risk unsystematic risk. The risk weighted alpha index is designed to be a long only or long/short index and is based to improve investments into stocks with increasing returns and low volatility. While, we have intended to develop the risk weighted alpha index as a passive indexation method, regardless, it is possible to use this method in high frequency or algorithmic trading as this method automatically calculates risk weighted alphas for each stock at every time the weights are recalibrated.

\subsection{Conclusion and policy implications}

This paper has introduced the risk weighted alpha (RWA) indexation methodology for index/portfolio construction. It argues that stocks with superior risk weighted alpha will have lower volatility and increasing returns. Risk weighted alpha is calculated as Jensen's Alpha divided by the standard deviation of the stock. Analyzing the stocks that comprise the ASX50 index and re-weighting these stocks based on the risk weighted alpha indexation method, it was seen that the risk weighted alpha index provided nearly five times the return with approximately the same systematic risk as the ASX50 index and it is less volatile than the treynor's square and appraisal ratio indexes through the duration of $2^{\text {nd }}$ January $2002-31^{\text {st }}$ December 2012.

\section{References}

Amenc N., Goltz F., Martellini L., Retkowsky L., 2010. Efficient Indexation: An Alternative to Cap-Weighted Indices. EDHEC-Risk Institute.

Basu A.K., Forbes B., 2013. Does fundamental indexation lead to better risk-adjusted returns? New evidence from Australian Securities Exchange. Accounting \& Finance, 53(3).

Benartzi S., Thaler R.H. 2001.Naive Diversification Strategies in Defined Contribution Saving Plans. American Economic Review, 91(1):79-98. http://dx.doi.org/10.1257/aer.91.1.79

Blitz D., Swinkels L., 2008. Fundamental indexation: An active value strategy in disguise. Journal of Asset Management, 9:264-269. http://dx.doi.org/10.1057/jam.2008.23

Blitz D., Bart van der G., van Vliet P. 2010. Fundamental Indexation: Rebalancing Assumptions and Performance. The Journal of Index Investing, 1(2):82-88. http://dx.doi.org/10.3905/jii.2010.1.2.082

Chia C.P., Melas D., Zhou T., 2011. Turbulent Times Ahead: Does Risk-Based Strategy Diversification Work? Journal of Indexes Europe.

Chow T., Hsu J.C., Kalesnik V., Little B., 2011. A Survey of Alternative Equity Index Strategies. Financial Analysts Journal, vol. 67(5):37-57. http://dx.doi.org/10.2469/faj.v67.n5.5

Choueifaty Y., Coignard Y., 2008. Toward Maximum Diversification. Journal of Portfolio Management, 35(1):40-51. http://dx.doi.org/10.3905/JPM.2008.35.1.40

Clarke R.G., de Silva H., Thorley S., 2006. Minimum-Variance Portfolios in the U.S. Equity Market. Journal of Portfolio Management, 33(1):10-24. http://dx.doi.org/10.3905/jpm.2006.661366

Demey P., Maillard S., Roncalli T., 2010. Risk-based Indexation. Working paper, Lyxor Asset Management.

DeMiguel V., Garlappi L. and Uppal R. 2009., Optimal Versus Naive Diversification: How Inefficient is the 1/N Portfolio Strategy? Review of Financial Studies, 22:1915-1953. http://dx.doi.org/10.1093/rfs/hhm075

Fernholz R., 1995. Portfolio Generating Functions. Working paper, INTECH.

Fernholz R., Garvy R., Hannon J., 1998. Diversity-Weighted Indexing. Journal of Portfolio Management, 24(2):74-82. http://dx.doi.org/10.3905/jpm.24.2.74

Haugen R.A., Baker N.L. 1991. The Efficient Market Inefficiency of Capitalization-Weighted Stock Portfolios. Journal of Portfolio Management, 17(3):35-40. http://dx.doi.org/10.3905/jpm.1991.409335

Hsu J.C., 2006. Cap-Weighted Portfolios Are Sub-Optimal Portfolios. Journal of Investment Management, 4(3):1-10.

Hsu J.C., Kalesnik V., Surti H., 2010. An Examination of Traditional Style Indices. Journal of Index Investing, 1(2):1423. http://dx.doi.org/10.3905/jii.2010.1.2.014 
Hsu J.C., Kalesnik V., Xie S. 2011. What Makes Fundamental Index Methodology Work? Working paper, Research Affiliates.

Kaplan P.D. 2008. Why Fundamental Indexation Might—or Might Not-Work. Financial Analysts Journal. 64(1):3239. http://dx.doi.org/10.2469/faj.v64.n1.5

Lowry V.T., 2007. Fundamentally Better. Journal of Indexes, (March/April):24-31. Mar J., Bird R., Casavecchia L. and Yeung D. Fundamental Indexation: An Australian Investigation. Australian Journal of Management. 34(1):1-20.

Markowitz H., 1952. Portfolio Selection. Journal of Finance, 7(1):77-99. http://dx.doi.org/10.1111/j.15406261.1952.tb01525.x

Markowitz H., 1959. Portfolio Selection: Efficient Diversification of Investments. Cowles Foundation Monograph No. 16. New York: John Wiley \&Sons, Inc.

Perold A.F., 2007. Fundamentally Flawed Indexing. Financial Analysts Journal, 63(6):31-37. http://dx.doi.org/10.2469/faj.v63.n6.4924

Robert A.D., Hsu J.C., 2008. Noise, CAPM and the Size and Value Effects. Journal of Investment Management, 6(1):111.

Robert A.D., Kalesnik V., Moghtader P., Scholl C., 2010. Beyond Cap Weight: The Empirical Evidence fora Diversified Beta. Journal of Indexes, 13(1):16-29.

Seigel J.J., 2006. The 'Noisy Market' Hypothesis. The Wall Street Journal (June 14): A14.

Sharpe W.F., 1964. Capital Asset Prices: A Theory of Market Equilibrium under Conditions of Risk. Journal of Finance, 19(3):425-442. http://dx.doi.org/10.1111/j.1540-6261.1964.tb02865.x

Waid R.J., 2007. Fundamentally Active. Working paper, Wilshire Associates.

Windcliff H., Boyle P., 2004. The 1/n Pension Plan Puzzle. North American Actuarial Journal, 8:32-45. http://dx.doi.org/10.1080/10920277.2004.10596151 